\title{
Succinate accumulation links mitochondrial MnSOD depletion to aberrant nuclear DNA methylation and altered cell fate
}

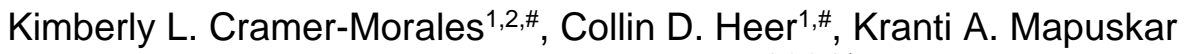 \\ and Frederick E. Domann ${ }^{1,2,3,4, *}$ \\ Departments of Radiation Oncology ${ }^{1}$, Surgery ${ }^{2}$, and Pathology ${ }^{3}$, Holden Comprehensive \\ Cancer Center ${ }^{4}$, The University of Iowa, Iowa City, lowa 52242 \\ \#These authors contributed equally \\ *Correspondence to frederick-domann@uiowa.edu
}

\begin{abstract}
Previous studies showed that human cell line HEK293 lacking mitochondrial superoxide dismutase (MnSOD) exhibited decreased succinate dehydrogenase (SDH) activity, and mice lacking MnSOD displayed significant reductions in SDH and aconitase activities.

Since MnSOD has significant effects on SDH activity, and succinate is a key regulator of TET enzymes needed for proper differentiation, we hypothesized that SOD2 loss would lead to succinate accumulation, inhibition of TET activity, and impaired erythroid precursor differentiation. To test this hypothesis, we genetically disrupted the SOD2 gene using the CRISPR/Cas9 genetic strategy in a human erythroleukemia cell line (HEL 92.1.7) capable of induced differentiation toward an erythroid phenotype. Cells obtained in this manner displayed significant inhibition of SDH activity and $\sim 10$-fold increases in cellular succinate levels compared to their parent cell controls. Furthermore, SOD2 ${ }^{-1-}$ cells exhibited significantly reduced TET enzyme activity concomitant with decreases in genomic 5-hmC and corresponding increases in 5-mC. Finally, when stimulated with $\delta$-aminolevulonic acid ( $\delta$-ALA), SOD2 $2^{-/-} \mathrm{HEL}$ cells failed to properly differentiate toward an erythroid phenotype, likely due to failure to complete the necessary global DNA demethylation program required for erythroid maturation. Together, our findings support the model of an SDH/succinate/TET axis and a role for succinate as a retrograde signaling molecule of mitochondrial origin that significantly perturbs nuclear epigenetic reprogramming and introduce MnSOD as a governor of the $\mathrm{SDH} /$ succinate/TET axis.
\end{abstract}

\section{Keywords}

Mitochondria, Superoxide Dismutase, iron homeostasis, Succinate Dehydrogenase, retrograde signaling, DNA methylation, epigenetic control, gene expression 


\section{Introduction}

Epigenetic processes provide a mechanism for integrating metabolic inputs and translating them into various phenotypic outputs [1,2]. Indeed, epigenetic modifiers often require or are inhibited by key citric acid cycle (CAC) metabolites (e.g. succinate, fumarate, alpha-ketoglutarate, acetyl-CoA) and co-factors (e.g. NAD $\left.{ }^{+}, F A D\right)$ [3]. One such metabolite, succinate, has recently been shown to act as a mitochondrial retrograde signal that exerts effects on the epigenome via inhibition of histone demethylases and the teneleven translocase (TET) family of DNA demethylase enzymes $[4,5]$.

TET family enzymes contribute to the maintenance of the epigenome by catalyzing the oxidation of 5-methylcytosine (5-mC) to 5-hydroxymethylcytosine (5-hmC), initiating a series of oxidation reactions necessary for the removal of 5-mC. Like other epigenetic modifiers, TET family enzymes are sensitive to metabolic perturbations affecting the absolute and relative concentrations of their co-factors and products. Their required cofactors and substrates are identical to those of the collagen and HIF1 proline hydroxylase (PHD) family of enzymes and include molecular oxygen $\left(\mathrm{O}_{2}\right)$, ferrous iron $\left(\mathrm{Fe}^{2+}\right)$, $\alpha$ ketoglutarate $(\alpha-K G)$, and ascorbate [6]. Using an Fe $\mathrm{Fe}^{\mathrm{IV}}$-oxo complex generated by the oxidative decarboxylation of $\alpha-K G$, TET enzymes catalyze the abstraction of a hydrogen from $5-\mathrm{mC}$, which is then hydroxylated. In the process, the oxidative decarboxylation of $\alpha$ $\mathrm{KG}$ yields succinate and carbon dioxide $\left(\mathrm{CO}_{2}\right)$ [7]. Through a product-induced negativefeedback mechanism, the inhibition of TET enzymes by succinate allows these enzymes to respond directly to changes in cellular metabolism, particularly to nodes linked to succinate and $\alpha-K G$ production and consumption.

Succinate and $\alpha-K G$ serve as key regulators of self-renewal and differentiation through TET enzymes. However, this regulation is complex and context-dependent $[8,9]$. For example, naïve embryonic stem cells sustain a high $\alpha-K G$ : succinate ratio to facilitate DNA and histone demethylation required for the maintenance of pluripotency $[10,11]$. However, $\alpha-K G$ is also important for the facilitation of epigenetic remodeling during differentiation of primed pluripotent stem cells [12] and premalignant cells in which p53 has been activated [13]. In the case of erythroid precursors, the model used in this study, global demethylation is required for the differentiation into red blood cells (RBCs) [14] and loss of TET enzymatic activity results in promoter hypermethylation and impaired differentiation of embryonic and hematopoietic stem cells $[12,15,16]$. Thus, the metabolic ratio of succinate to $\alpha-K G$ is a key factor in determining self-renewal and differentiation through modulation of TET activity.

A primary regulator of succinate levels is the activity of succinate dehydrogenase $(\mathrm{SDH}) . \mathrm{SDH}$ is a 4-subunit complex located in the inner membrane of the mitochondria that plays a role in the CAC via catalysis of the oxidation of succinate to fumarate as well as serving the role of Complex II in the electron transport chain by passing electrons from succinate or $\mathrm{FADH}_{2}$ directly to coenzyme $\mathrm{Q}$ in the mitochondrial inner membrane. Pharmacological inhibition or genetic mutation of SDH leads to accumulation of succinate and inhibition of $\alpha-K G$-dependent enzymes such as TETs [5,17-19]. SDH activity can also be modulated by reactive oxygen species (ROS). Specifically, superoxide $\left(\mathrm{O}_{2}{ }^{-*}\right)$ can inactivate iron-sulfur (Fe-S) cluster-containing enzymes such as SDH [18,19], as well as other enzymes in the CAC (aconitase, fumarate hydratase) [20,22,23], heme synthesis (ferrochelatase; FECH)[24], and amino acid metabolism [25,26] among several others.

Manganese superoxide dismutase (MnSOD) catalyzes the dismutation of mitochondrial $\mathrm{O}_{2}{ }^{*-}$ to hydrogen peroxide $\left(\mathrm{H}_{2} \mathrm{O}_{2}\right)$. Cells lacking MnSOD exhibit elevated steady state levels of $\mathrm{O}_{2}{ }^{*-}$ and reduced $\mathrm{SDH}$ activity, likely due to inactivation of Fe-S 
clusters [20,27-29]. Since succinate is a direct regulator TET enzymatic activity and MnSOD has significant effects on SDH activity, we hypothesized that MnSOD loss would lead to succinate accumulation, inhibition of TET enzyme activity and altered cellular phenotype. To test this hypothesis, we used the CRISPR/Cas9 strategy to genetically disrupt the SOD2 gene in two different human cell lines, human embryonic kidney 293 (HEK293) generated in a previous study [28], and a human erythroleukemia (HEL) generated in this study, thereby eliminating MnSOD activity. We chose HEL cells as a model because RBC maturation to produce mature enucleated erythrocytes requires rapid and widespread demethylation of genomic DNA [14] and these cells can be induced to differentiate toward mature red cells upon supplementation with $\delta$-aminolevulinic acid $(\delta$ ALA). Thus, we could utilize this model to study the effects of MnSOD loss on DNA demethylation-dependent erythroid differentiation.

Following characterization of the genetically disrupted SOD2 cell lines, we measured the activities of mitochondrial enzymes sensitive to inactivation by $\mathrm{O}_{2}{ }^{*-}$ (SDH, aconitase), succinate levels, TET enzyme activity, TET substrate (5-mC) and product (5hmC) levels, and ability of HEL cells to undergo erythroid maturation in response to $\delta$-ALA exposure. We found that MnSOD loss resulted in decreased SDH activity, succinate accumulation, reduced activity of TET enzymes, inhibition of DNA demethylation, and impaired erythroid differentiation. Taken together, our results introduce a mechanism through which MnSOD exerts epigenetic control over differentiation through an MnSOD/SDH/succinate/TET axis, and further support a role for succinate as a retrograde signaling molecule of mitochondrial origin.

\section{Materials and Methods}

\section{Cell Lines}

HEK293T cells were obtained from American Type Culture Collection (ATCC) maintained in DMEM supplemented with $10 \%$ fetal bovine serum in a humidified $37^{\circ} \mathrm{C}$ incubator with $5 \% \mathrm{CO}_{2}$ and were routinely sub-cultured before reaching confluence by detachment with TrypLE Express (Invitrogen, Carlsbad, CA).

HEL 92.1.7 cells were obtained from ATCC and cultured in RPMI640 supplemented with $10 \%$ fetal bovine serum in a humidified $37^{\circ} \mathrm{C}$ incubator with $5 \% \mathrm{CO}_{2}$ and were routinely sub-cultured by 1:10 dilutions in fresh media.

\section{Plasmid Construction and Transfection}

The pD130-GFP expression vector was designed to contain expression cassettes for green fluorescent protein (GFP), Cas9 endonuclease, and a CRISPR chimeric cDNA with the gRNA moiety designed to target exon 3 of SOD2 [ATATCAATCATAGCATTTTC]. This plasmid was custom synthesized (DNA2.0, Menlo Park, CA) and following amplification, purification, and characterization was used to transfect the two cell lines described above.

HEK293T cells were transiently transfected by calcium phosphate precipitation. Five days after transfection GFP+ cells were sorted and collected by flow cytometry and colonies were selected from single clones using cloning discs.

HEL 92.1.7 cells were transfected by electroporation at various conditions using the Gene Pulser Xcell ${ }^{\mathrm{TM}}$ Electroporation System (BioRad, USA). In brief, $5 \times 10^{6}$ cells were suspended in $500 \mu \mathrm{l}$ complete RPMI medium, mixed with $30 \mu \mathrm{g}$ of pD130-GFP-plasmid in 
a $4 \mathrm{~mm}$ electroporation cuvette, and incubated on ice for $10 \mathrm{~min}$. After electroporation with a single pulse (electrical parameters of $300 \mathrm{~V}, 950 \mu \mathrm{F}$ ) the cells were transferred into complete RPMI medium at a density of $1 \times 10^{6}$ cells per $\mathrm{ml}$ and returned to the incubator. Five days after transfection GFP+ cells were sorted by flow cytometry and colonies were selected from single clones seeded in 96-well plates.

\section{Western Blot Analyses}

Western blots were performed as previously described [26]. Briefly, total proteins were extracted from HEL 92.1.7 clones with standard RIPA buffer plus protease inhibitors and quantified by Bradford assay. Proteins were separated by SDS-PAGE, transferred to nitrocellulose membranes and probed with primary antibodies detecting MnSOD (Cat \# 06-984, Millipore, Billerica, MA) and beta-tubulin (University of lowa Hybridoma Core, lowa City, IA).

\section{Aconitase activity}

Aconitase activity was measured as described previously [30]. In brief, in a spectrophotometric assay where total cellular protein was combined with $\mathrm{NADP}^{+}$and isocitrate dehydrogenase and the appearance of NADPH was monitored at $340 \mathrm{~nm}$ every 30s for 5 minutes.

\section{Succinate Dehydrogenase Histochemistry}

Succinate dehydrogenase activity visualized via NBT reduction histochemistry. Culture medium was aspirated and cells were washed twice with PBS. Cell culture dishes were allowed to air dry at room temperature. A solution containing $0.55 \mathrm{mM} \mathrm{NBT}$ and $0.05 \mathrm{M}$ sodium succinate was applied and the dishes were incubated overnight at $37^{\circ} \mathrm{C}$ and washed again with PBS before images were captured.

\section{Determination of Succinate levels}

Succinate levels were determined using a colorimetric assay (Sigma-Aldrich Cat \# MAK184) according to the manufacturer's specifications.

\section{Tet DNA Demethylase Activity and 5-Hydroxy-Methylcytosine (5hmC) Measurement}

Cellular TET DNA demethylase activities were assessed using the EpiGentek TET activity assay platform. Total genomic 5-methylcytosine and 5-hydroxymethylcytosine levels were measured in genomic DNA with the corresponding immunoassay systems also from EpiGentek according to the manufacturer's instructions.

\section{Flow Cytometry Analysis}

Determinations were performed with a flow cytometer (FACScan, Becton Dickinson, San Jose, CA). HEK293T or HEL 92.1.7 cells were gated on the basis of their forward scatter and side scatter signals with logarithmic scales. The region representing single, nonaggregated cells was gated before each experience with non-agglutinated samples to count events in the single-cell region. Fluorescence profiles were collected only on those cells appearing in this region to exclude agglutinates and debris. For each sample, 20,000 
events were collected in logarithmic scale. Photomultiplier tube voltage for the fluorescence detector was adjusted before running samples to obtain a negative fluorescence signal for control (untransfected) samples. This source power value was fixed for all measurements. Computer software (WinMDI, version 2.8, Windows multiple document interface, flow cytometry application, Microsoft, Redmond, WA) was used for data analysis to calculate mean fluorescence intensity (MFI) from fluorescence histograms.

Antibodies utilized were directed against TFRC (PE-tagged anti-CD71 clone M-A712, BD Biosciences, San Jose, CA) and Glycophorin A (PE-tagged-anti-CD235a clone REA175, Miltenyi Biotech, Auburn, CA), markers of human erythroid maturation. Cells were incubated with antibodies under conditions previously described [31-33] and flow cytometry was performed as described above.

\section{Results}

\section{Characterization of MnSOD loss in CRISPR/Cas9-edited HEL 92.1.7 cells}

The CRISPR/Cas9 system for creating specific genetic deletions was used to target exon 3 of human SOD2 in a HEL 92.1.7 cells. SOD2/- HEK293T were generated previously [28]. Two clones from each cell line were confirmed to show absence of MnSOD protein expression by Western blot analysis (Figure 1) [28]. In a previous study, we showed that MnSOD-deficient 293T $\left(S O D 2^{-/}\right)$cells showed significantly increased DHE and MitoSOX fluorescence compared to wild-type (WT) cells, suggesting increased cytosolic and mitochondrial pro-oxidants levels, respectively [28].

SOD2 KO cells exhibited decreased activity in enzymes sensitive to inactivation by $\mathrm{O}_{2}{ }^{-*}$ and accumulation of cellular succinate.

Having established and characterized suitable model systems, we next sought to determine if SOD2 ${ }^{-/}$cells exhibited decreased activity in mitochondrial enzymes known to be sensitive to inactivation by $\mathrm{O}_{2}{ }^{\circ}$. Aconitase and $\mathrm{SDH} / \mathrm{Complex}$ II are key players in mitochondrial metabolism that contain Fe-S clusters susceptible to inactivation by $\mathrm{O}_{2}{ }^{-*}$ [20-22]. Aconitase activity was measured in a spectrophotometric assay where total cellular protein was combined with $\mathrm{NADP}^{+}$and isocitrate dehydrogenase and the appearance of NADPH was measured at $340 \mathrm{~nm}$. As expected, we observed a significant decrease in aconitase activity in SOD2 $2^{-/} \mathrm{HEL}$ cells, likely due to inactivation by increased steady state levels of $\mathrm{O}_{2}{ }^{--}$(Figure 2A). In addition, SDH activity was significantly decreased in SOD2 ${ }^{-/-}$HEL cells (Figure 2B) as was also observed in SOD2 $2^{-/}$HEK293 cells in a previous study [28]. Consistent with the observed decrease in SDH activity, cellular succinate levels increased $\sim 5$-fold in both 293T and HEL KO cells (Figure 2C). Taken together, these results indicate that MnSOD loss is associated with significantly decreased SDH activity, likely through $\mathrm{O}_{2}{ }^{*-}$-mediated disruption of Fe-S clusters in SDH subunit $\mathrm{B}$, and that loss of SDH activity is co-incident with increased levels of cellular succinate, the substrate for $\mathrm{SDH}$.

Activity of TET family of demethylation enzymes was diminished in cells without MnSOD expression

TET DNA demethylases are nuclear enzymes that utilize $\alpha-K G$ and generate succinate, by which they known to be product inhibited. Therefore, we next sought to 
determine if the accumulation of succinate that occurs in SOD2 $2^{--}$cells coincided with decreased activity of TET family enzymes. To this end, TET activity was measured in an antibody-mediated colorimetric assay using nuclear protein. The results of this assay revealed a $\sim 2$-fold reduction in TET family activity in both SOD2-/ 293T cells (blue striped bar) and SOD2 ${ }^{--}$HEL cells (red striped bar) (Figure 3) compared to their WT parent cell types. Overall, these results demonstrate a clear association between loss of MnSOD expression and decreased activity of TET demethylase enzymes, likely due to productinduced negative feedback inhibition resulting from elevated succinate levels.

\section{TET enzyme inactivation disrupted epigenetic programming and altered cell fate}

Based on the observation of decreased TET enzyme activity in SOD2 $2^{-/}$cell lines, we hypothesized that this would constitute a DNA demethylation defect and that these cells would therefore display an increase in the percentage of genomic 5-mC. Indeed, we observed a statistically significant increase in percent 5-mC in both 293T and HEL SOD2/ cells compared to their respective WT parent cell lines (Figure 4A). In parallel, we observed a corresponding decrease in the percentage of the TET enzyme product 5-hmC in HEL cells lacking MnSOD expression (Figure 4B). These results agree well with the finding of decreased TET enzyme activity and demonstrate that decreased TET enzyme activity is associated with an increase in TET enzyme substrate (5-mC) and decrease in TET enzyme product (5-hmC) potentially contributing to dysregulated homeostasis of DNA methylation

The process of erythroid maturation is dependent on global DNA demethylation, which we demonstrated was disrupted in SOD2 $2^{-/}$cells. To determine whether the DNA demethylation defect in SOD2 ${ }^{-/}$cells would impair the ability of erythroid-like precursor cells to differentiate toward RBCs, we compared the ability of parent WT HEL cells to SOD2 $\%$ HEL cells to differentiate upon exposure to $\delta$-aminolevulinic acid ( $\delta$-ALA). Traditional markers (CD235a/Glycophorin A and CD71/Transferrin receptor) were used to assess erythroid maturation of HEL cells by flow cytometry. Differentiating RBC precursors display a wave of elevated CD71 expression that diminishes upon complete maturation to RBCs, and mature human erythroid cells are CD235a-positive and CD71-negative [34,35]. At baseline, SOD2 ${ }^{-/}$cells exhibited slightly higher CD71 and CD235a expression compared to WT cells.

To initiate erythroid maturation, HEL cells were grown in the presence or absence of $100 \mu \mathrm{M} \delta$-aminolevulinic acid ( $\delta$-ALA) for 5 days. After incubation with $\delta$-ALA for 5 days, WT HEL cells exhibited relatively low CD71 surface expression, consistent with a mature CD71-negative phenotype (Figure 5, top). By contrast, HEL SOD2 ${ }^{-/}$cells (clone 5, blue and clone 13, orange) exhibited 70-80 fold increases in CD71 surface expression upon treatment with ALA demonstrating a significant defect in their ability to downregulate CD71 upon completion of the RBC maturation process (Figure 5, top). In the case of Glycophorin/CD235a, parent WT HEL cells upregulated CD235a 4-fold upon exposure to $\delta$-ALA, consistent with normal erythroid maturation (Figure 5, bottom). In contrast, SOD21- HEL cells exhibited a 2-4 fold decrease in CD235a (Figure 5 bottom). These results are quantified in Table 1. After 5 days in ALA, parent WT HEL cells resemble maturing erythroid cells (low TFRC, increased GlyA) whereas SOD2 ${ }^{-/}$cells displayed aberrant expression of these biomarkers (high TFRC, decreased GlyA), consistent with their defect in DNA demethylation which is required for proper erythroid differentiation. Thus, SOD2 ${ }^{-/-}$ HEL cells fail to display proper erythroid maturation. We propose that this impairment is likely due to a deficiency in TET-driven DNA demethylation. 


\section{Discussion}

We tested the overarching hypothesis that succinate serves as a mitochondrial retrograde signal linking MnSOD activity to nuclear epigenetic reprogramming. Our results suggest a model in which increased mitochondrial $\mathrm{O}_{2}{ }^{*-}$ levels in cells lacking MnSOD leads to inhibition of SDH activity and accumulation of succinate. In turn, this build-up of succinate causes inactivation of the TET family of DNA demethylases. Such disruption of epigenetic homeostasis might contribute to aberrations in developmental pathways or maintenance of cell-type lineage stability. While in this study we have used erythroid differentiation in SOD2 ${ }^{-/}$HEL cells as a phenotypic indicator of disrupted DNA demethylase activity, we also showed disrupted DNA demethylase activity and 5-mC levels in HEK293 cells, thus demonstrating that this effect is not specific to a single cell line or cell type. This data supports the model of an SDH/succinate/TET axis and a role for succinate as a retrograde signaling molecule of mitochondrial origin that significantly perturbs nuclear epigenetic reprogramming to affect cellular phenotype or differentiation state (Figure 6). Furthermore, our findings introduce MnSOD as a regulator of the $\mathrm{SDH} /$ succinate/TET axis and propose that SDH inactivation in the context of SOD2 knockout is a result of Fe-S cluster inactivation caused by increased steady-state levels of $\mathrm{O}_{2}{ }^{-*}$.

Of particular interest will be the continued investigation of the MnSOD/SDH/succinate/TET axis in the context of cancer progression and dedifferentiation. The MnSOD promoter contains a CpG island that is frequently methylated in cancer cells, and MnSOD activity decreases during early carcinogenesis [36-39]. Based on this knowledge, we have proposed an "epigenetic flywheel" in which loss of SOD activity contributes to epigenetic changes, leading to further CpG island hypermethylation of the promoters of SOD2 and tumor suppressor genes [40]. Findings in this study support the theory that decreased MnSOD activity is linked with increased DNA methylation.

Hypothetically, the epigenetic dysregulation caused by this "flywheel" effect could be initiated at any constituent of the MnSOD/SDH/TET axis. One set of initiators includes $\mathrm{SDH}$ mutations. Indeed, SDH mutations, are common in gastrointestinal stromal tumors and paragangliomas and are associated with decreased TET enzyme activity, DNA hypermethylation, poor differentiation, and occurrence of epithelial-to-mesenchymal transition (EMT) [17,41-45]. In addition to genetic mutations, SDH activity can be impaired by cellular iron deficiency induced by various stressors, such as lysosomal dysfunction $[46,47]$.

Relatedly, mutations to fumarate hydratase $(\mathrm{FH})$, another Fe-S cluster-containing protein, or isocitrate dehydrogenase (IDH), lead to accumulation of fumarate and D-2hydroxyglutarate, respectively, which act in a manner similar to succinate by inhibiting TET and Jumonji histone demethylases, leading to DNA and histone hypermethylation, impairment of homologous recombination [48-50] and failure to differentiate in several contexts [5,51-53]. Interestingly, SDH and IDH mutations alone are sufficient to activate oncogenic programs in the absence of classical kinase mutations by disrupting normal chromosomal topology [54,55].

Another potential set of initiators of the proposed "flywheel" include direct mutations to TET enzymes or differential bioavailability of TET enzyme substrates and cofactors. For example, genetic loss of TET enzymes can lead to increased hematopoietic stem cell self-renewal and eventual development of a myeloproliferative phenotype [56]. Regarding TET substrates, tumor hypoxia has been shown to induce hypermethylation via inhibition of TET [57] and $\mathrm{O}_{2}$ tension determines cellular differentiation by regulating 
TET activity in eSCs [58]. Availability of cofactors including $\mathrm{Fe}^{2+}$ and ascorbate have also been shown to regulate TET enzyme activity [59-61]. Some of this knowledge is already being translated into cancer therapies. For example, ascorbate treatment has been shown to increase TET enzymatic activity and 5-hmC levels and is associated with reduced tumor progression and/orgrowth in several contexts [62-64]. It should be noted that, while we did not examine the effect of succinate accumulation of histone demethylase activity in this study, we postulate that aberrant histone methylation may also be occurring under these conditions, as KDMs are also $\alpha-K G$ dependent dioxygenases amenable to inhibition by succinate. Such hypotheses will be the focus to future studies.

Ultimately, our results present a link between mitochondrial oxidative metabolism and epigenetic regulation, support the role of succinate in the growing list of metabolic regulators of the epigenome, and introduce MnSOD as a modulator of succinate levels and TET enzyme activity, likely through control of SDH activity.

\section{Acknwlwdgements}

This work was supported by NIH grants R01CA115438, P30CA086862, T32CA078586, and P01CA217797. 


\section{Figure Legends}

Figure 1. Characterization of MnSOD loss in CRISPR/Cas9-edited HEL 92.1.7 cells. The CRISPR/Cas9 system for creating specific genetic deletions was used to target exon 3 of human SOD2 in a human erythroleukemia cell line (HEL 92.1.7). Total protein was isolated from clones transfected with the CRISPR/Cas9 expression construct and subjected to Western blot analysis. Two clones were confirmed to show absence of MnSOD protein expression by Western blot analysis.

Figure 2. SOD2 KO cells exhibit decreased activity in enzymes sensitive to inactivation by superoxide and an accumulation of cellular succinate. A) Aconitase activity was measured in a spectrophotometric assay where total cellular protein was combined with $\mathrm{NADP}^{+}$and isocitrate dehydrogenase and the appearance of NADPH was monitored every 30s for 5 minutes. B) Succinate dehydrogenase activity visualized via NBT reduction histochemistry was significantly reduced in both 293T and HEL cells without MnSOD expression. C) Total cellular succinate increased $\sim 5$-fold in both 293T and HEL KO cells. * $p<.01$ compared to WT.

Figure 3. Activity of TET family of demethylation enzymes is diminished in cells lacking MnSOD. TET activity was measured in an antibody-mediated colorimetric assay using nuclear protein. A -2-fold reduction in TET family activity was observed in MnSOD- deficient 293T cells (blue striped bar) and HEL cells (red striped bar). $p<.05$ compared to WT.

Figure 4. TET enzyme inactivation is associated with perturbed global epigenetic programming. A) An expected increase in the percentage of genomic 5-methylcytosine was observed in 293T and HEL KO cells. B) A corresponding decrease in the percentage of genomic 5-hydroxymethylcytosine was detected in 293T and HEL cells lacking MnSOD expression. ${ }^{*} p<.01$ compared to WT.

Figure 5. Erythroid maturation is impaired in HEL KO cells compared to WT. With the addition of $\delta$-ALA, HEL KO cells (clone 5, blue and clone 13, orange) failed to downregulate expression of Transferrin receptor (TFRC) and decreased expression of Glycophorin A (GlyA) compared to WT cells (red) as determined by inspection of AUC.

Figure 6. Model depicting a central role for loss of MnSOD in disrupting differentiation. Loss of MnSOD leads to higher mitochondrial superoxide which disrupts mitochondrial non-heme iron proteins including SDH resulting in elevated cellular succinate and product-inhibition of $\alpha-K G$ dependent TET enzymes, resulting in large scale alterations in genomic DNA methylation. 
HEL 92.1.7

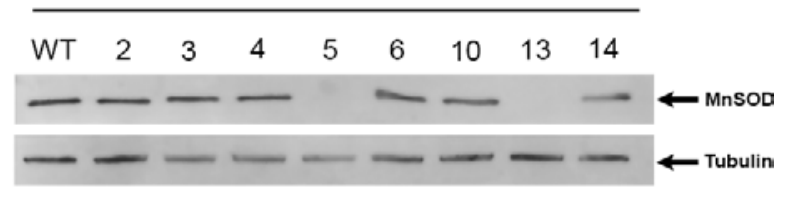

Figure 1. Characterization of MnSOD loss in CRISPR/Cas9-edited HEL 92.1.7 cells
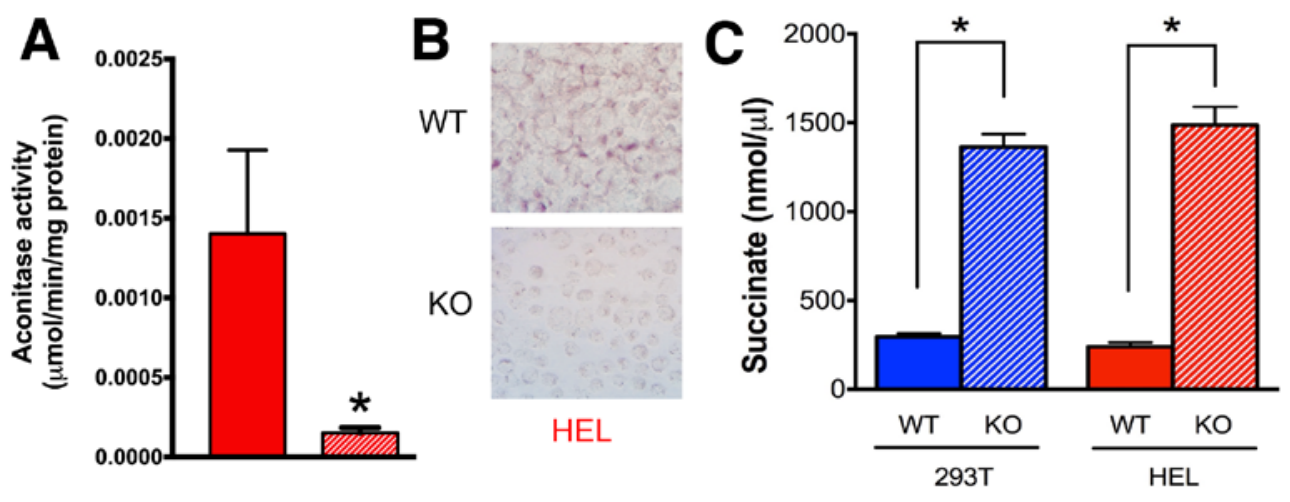

Figure 2. SOD2 KO cells exhibit decreased activity in enzymes sensitive to inactivation by superoxide and an accumulation of cellular succinate.

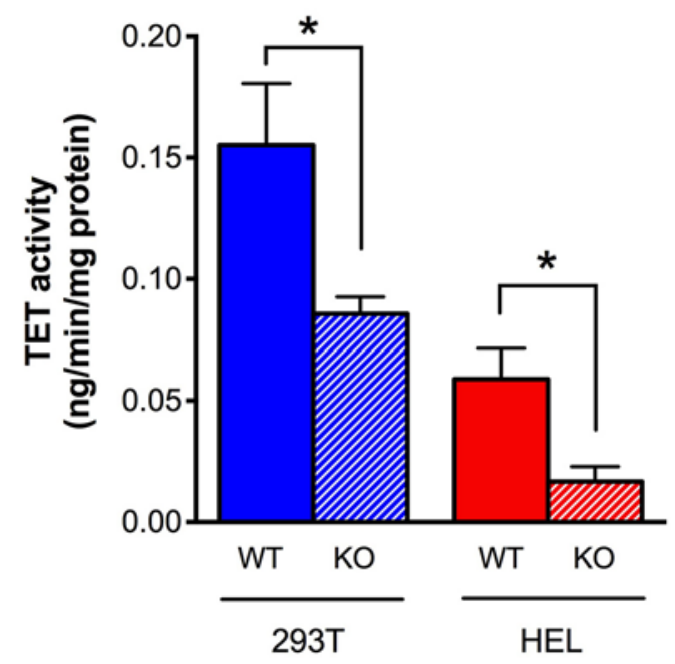

Figure 3. Activity of TET family of demethylation enzymes is diminished in cells without MnSOD expression. 
A

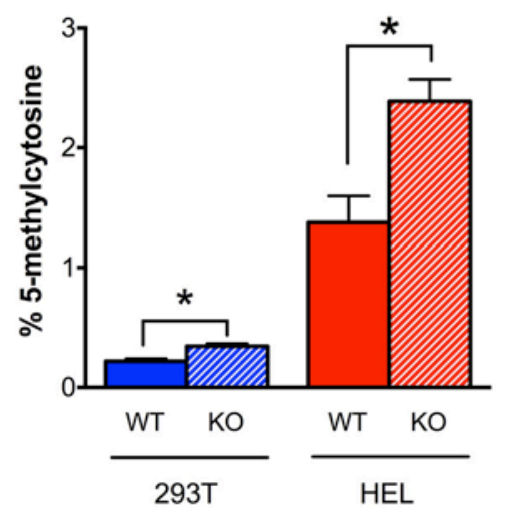

B

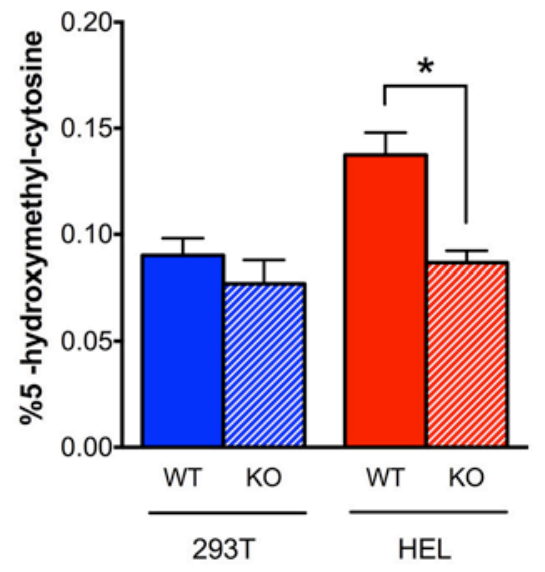

Figure 4. TET enzyme inactivation subsequently perturbs global epigenetic programming.
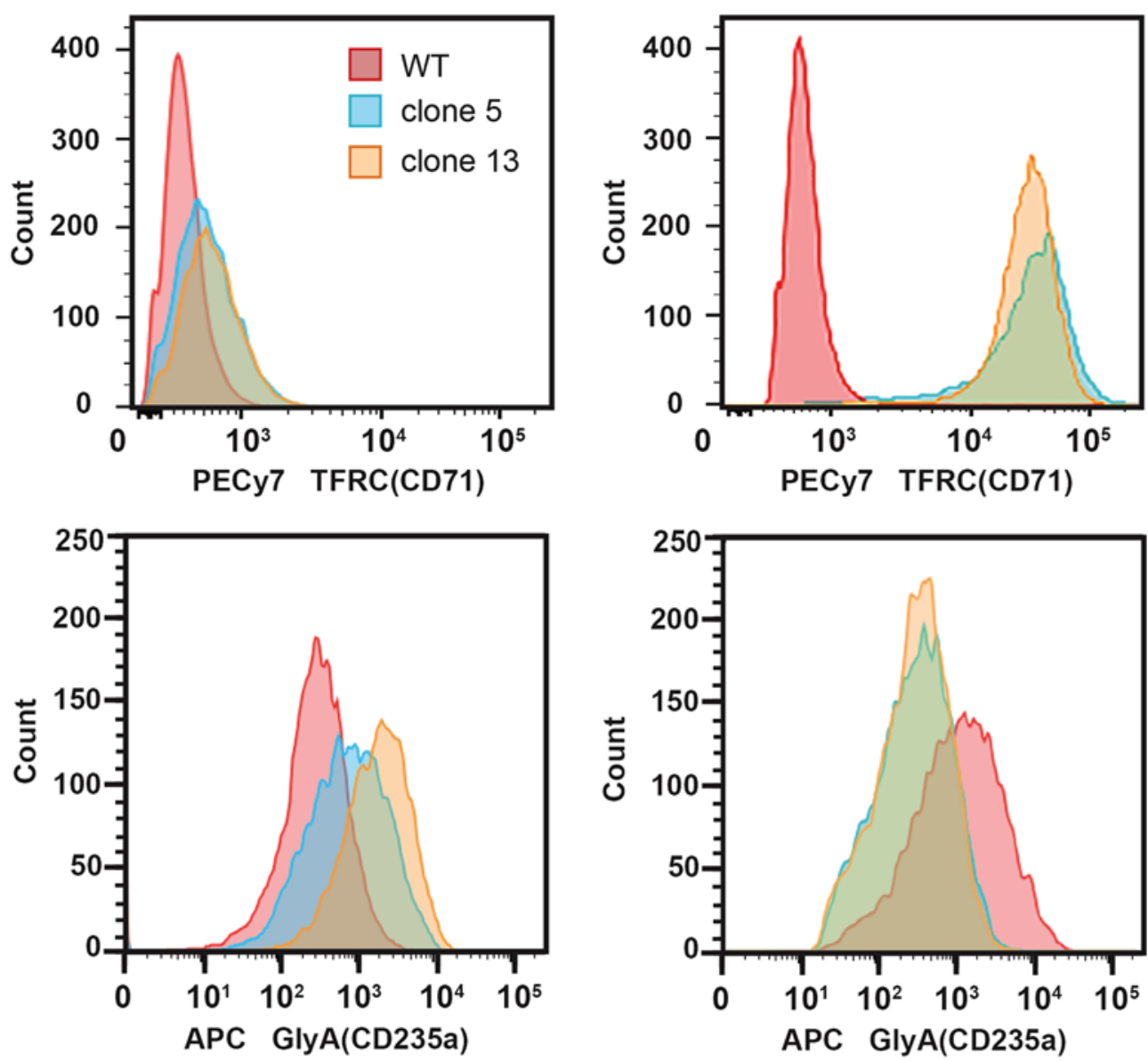

Figure 5. Erythroid maturation is impaired in HEL KO cells compared to WT. 
Table 1. MnSOD deficiency inhibits erythroid differentiation in response to $\delta A L A$. Mean fluorescence intensities (MFI) for surface expression of markers of erythroid differentiation, transferrin receptor (CD71) and glycophorin A (CD225a). After 5 days in $\delta A L A$, wild type HEL cells resemble maturing erythroid cells (low TFRC, high GlyA) whereas SOD2-- cells displayed aberrant expression of these biomarkers, consistent with their defect in DNA demethylation which is required for erythroid differentiation. AU - arbitrary units

\begin{tabular}{|l|c|c|c|}
\hline$\left(\mathrm{MFI}, \mathrm{AU} \times 10^{-2}\right)$ & $-\delta A L A$ & $+\delta A L A$ & Fold $\Delta$ \\
\hline & \multicolumn{3}{|c|}{ TFRC } \\
\hline WT HEL & 2.5 & 5.7 & 2.3 \\
\hline SOD2/- $\mathrm{cl} .5$ & 4.4 & 31 & 70 \\
\hline SOD2/- $\mathrm{cl} .13$ & 5.0 & 40 & 80 \\
\hline & \multicolumn{3}{|c|}{ Glycophorin A } \\
\hline WT HEL & 3.2 & 12 & 3.8 \\
\hline SOD2/- cl.5 & 9.0 & 4.5 & -2.0 \\
\hline SOD2 $2^{-/-} \mathrm{cl} .13$ & 20 & 4.5 & -4.4 \\
\hline
\end{tabular}




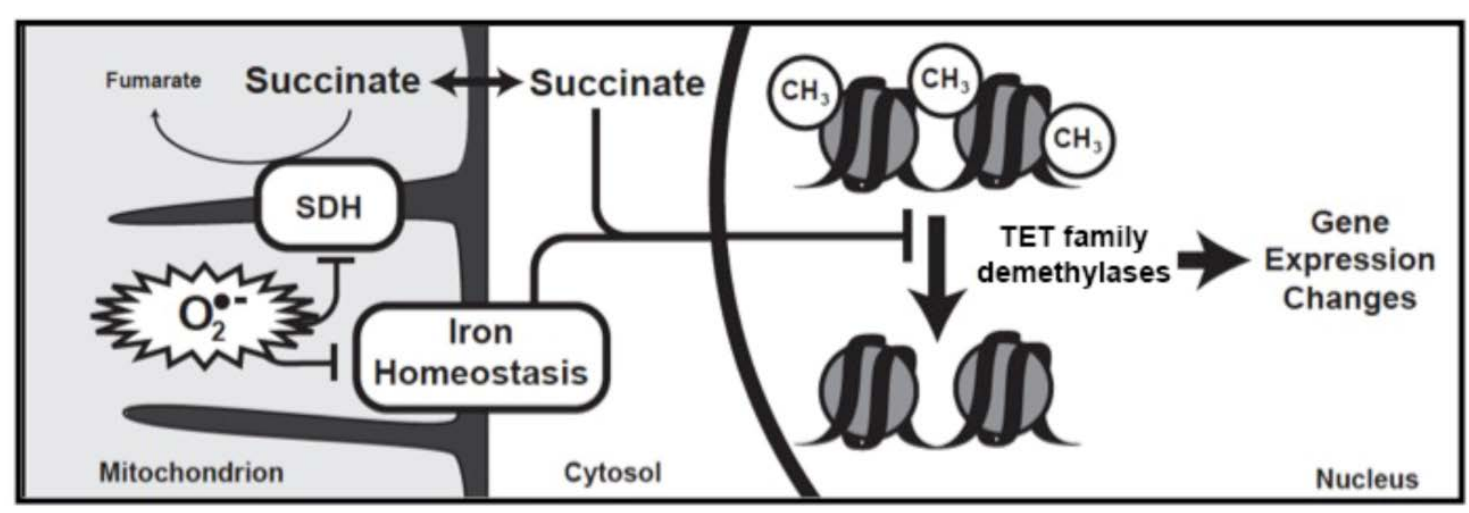

Figure 6. Model depicting a central role for loss of MnSOD leading to higher mitochondrial superoxide which disrupts non-heme iron proteins including SDH resulting in elevated cellular succinate and product-inhibition of $\alpha$-KG dependent TET enzymes. 


\section{References}

1. Cyr, A.R.; Domann, F.E. The Redox Basis of Epigenetic Modifications: From Mechanisms to Functional Consequences. Antioxid. Redox Signal. 2011, 15, 551-589, doi:10.1089/ars.2010.3492.

2. Janssen, J.J.E.; Grefte, S.; Keijer, J.; de Boer, V.C.J. Mito-Nuclear Communication by Mitochondrial Metabolites and Its Regulation by B-Vitamins. Front. Physiol. 2019, 10, doi:10.3389/fphys.2019.00078.

3. Hitchler, M.J.; Domann, F.E. An epigenetic perspective on the free radical theory of development. Free Radic. Biol. Med. 2007, 43, 1023-1036, doi:10.1016/j.freeradbiomed.2007.06.027.

4. Laukka, T.; Mariani, C.J.; Ihantola, T.; Cao, J.Z.; Hokkanen, J.; Kaelin, W.G.; Godley, L.A.; Koivunen, P. Fumarate and Succinate Regulate Expression of Hypoxiainducible Genes via TET Enzymes. J. Biol. Chem. 2016, 291, 4256-4265, doi:10.1074/jbc.M115.688762.

5. Xiao, M.; Yang, H.; Xu, W.; Ma, S.; Lin, H.; Zhu, H.; Liu, L.; Liu, Y.; Yang, C.; Xu, Y.; et al. Inhibition of $\alpha$-KG-dependent histone and DNA demethylases by fumarate and succinate that are accumulated in mutations of FH and SDH tumor suppressors. Genes Dev. 2012, 26, 1326-1338, doi:10.1101/gad.191056.112.

6. Kaelin, W.G. Cancer and Altered Metabolism: Potential Importance of HypoxiaInducible Factor and 2-Oxoglutarate-Dependent Dioxygenases. Cold Spring Harb. Symp. Quant. Biol. 2011, 76, 335-345, doi:10.1101/sqb.2011.76.010975.

7. Simmons, J.M.; Müller, T.A.; Hausinger, R.P. FeII/ $\alpha$-ketoglutarate hydroxylases involved in nucleobase, nucleoside, nucleotide, and chromatin metabolism. Dalton Trans. Camb. Engl. 2003 2008, 5132-5142, doi:10.1039/b803512a.

8. Tran, K.A.; Dillingham, C.M.; Sridharan, R. The role of $\alpha$-ketoglutaratedependent proteins in pluripotency acquisition and maintenance. J. Biol. Chem. 2019, 294, 5408-5419, doi:10.1074/jbc.TM118.000831.

9. Lees, J.G.; Gardner, D.K.; Harvey, A.J. Pluripotent Stem Cell Metabolism and Mitochondria: Beyond ATP Available online: https://www.hindawi.com/journals/sci/2017/2874283/ (accessed on Nov 2, 2020).

10. Carey, B.W.; Finley, L.W.S.; Cross, J.R.; Allis, C.D.; Thompson, C.B. Intracellular $\alpha-$ ketoglutarate maintains the pluripotency of embryonic stem cells. Nature 2015, 518, 413-416, doi:10.1038/nature13981.

11. Hwang, I.-Y.; Kwak, S.; Lee, S.; Kim, H.; Lee, S.E.; Kim, J.-H.; Kim, Y.A.; Jeon, Y.K.; Chung, D.H.; Jin, X.; et al. Psat1-Dependent Fluctuations in $\alpha$-Ketoglutarate Affect the Timing of ESC Differentiation. Cell Metab. 2016, 24, 494-501, doi:10.1016/j.cmet.2016.06.014.

12. TeSlaa, T.; Chaikovsky, A.C.; Lipchina, I.; Escobar, S.L.; Hochedlinger, K.; Huang, J.; Graeber, T.G.; Braas, D.; Teitell, M.A. $\alpha$-Ketoglutarate Accelerates the Initial Differentiation of Primed Human Pluripotent Stem Cells. Cell Metab. 2016, 24, 485-493, doi:10.1016/j.cmet.2016.07.002.

13. Morris, J.P.; Yashinskie, J.J.; Koche, R.; Chandwani, R.; Tian, S.; Chen, C.-C.; Baslan, T.; Marinkovic, Z.S.; Sánchez-Rivera, F.J.; Leach, S.D.; et al. $\alpha-$ 
Ketoglutarate links p53 to cell fate during tumour suppression. Nature 2019, 573, 595-599, doi:10.1038/s41586-019-1577-5.

14. Shearstone, J.R.; Pop, R.; Bock, C.; Boyle, P.; Meissner, A.; Socolovsky, M. Global DNA Demethylation During Mouse Erythropoiesis in Vivo. Science 2011, 334, 799-802, doi:10.1126/science.1207306.

15. Dawlaty, M.M.; Breiling, A.; Le, T.; Barrasa, M.I.; Raddatz, G.; Gao, Q.; Powell, B.E.; Cheng, A.W.; Faull, K.F.; Lyko, F.; et al. Loss of Tet Enzymes Compromises Proper Differentiation of Embryonic Stem Cells. Dev. Cell 2014, 29, 102-111, doi:10.1016/j.devcel.2014.03.003.

16. Ko, M.; Bandukwala, H.S.; An, J.; Lamperti, E.D.; Thompson, E.C.; Hastie, R.; Tsangaratou, A.; Rajewsky, K.; Koralov, S.B.; Rao, A. Ten-Eleven-Translocation 2 (TET2) negatively regulates homeostasis and differentiation of hematopoietic stem cells in mice. Proc. Natl. Acad. Sci. 2011, 108, 14566-14571, doi:10.1073/pnas.1112317108.

17. Letouzé, E.; Martinelli, C.; Loriot, C.; Burnichon, N.; Abermil, N.; Ottolenghi, C.; Janin, M.; Menara, M.; Nguyen, A.T.; Benit, P.; et al. SDH Mutations Establish a Hypermethylator Phenotype in Paraganglioma. Cancer Cell 2013, 23, 739-752, doi:10.1016/j.ccr.2013.04.018.

18. Cordes, T.; Wallace, M.; Michelucci, A.; Divakaruni, A.S.; Sapcariu, S.C.; Sousa, C.; Koseki, H.; Cabrales, P.; Murphy, A.N.; Hiller, K.; et al. Immunoresponsive Gene 1 and Itaconate Inhibit Succinate Dehydrogenase to Modulate Intracellular Succinate Levels. J. Biol. Chem. 2016, 291, 14274-14284, doi:10.1074/jbc.M115.685792.

19. Hoekstra, A.S.; de Graaff, M.A.; Briaire-de Bruijn, I.H.; Ras, C.; Seifar, R.M.; van Minderhout, I.; Cornelisse, C.J.; Hogendoorn, P.C.W.; Breuning, M.H.; Suijker, J.; et al. Inactivation of SDH and FH cause loss of $5 \mathrm{hmC}$ and increased H3K9me3 in paraganglioma/pheochromocytoma and smooth muscle tumors. Oncotarget 2015, 6, 38777-38788.

20. Powell, C.S.; Jackson, R.M. Mitochondrial complex I, aconitase, and succinate dehydrogenase during hypoxia-reoxygenation: modulation of enzyme activities by MnSOD. Am. J. Physiol.-Lung Cell. Mol. Physiol. 2003, 285, L189L198, doi:10.1152/ajplung.00253.2002.

21. Zhang, Y.; Marcillat, O.; Giulivi, C.; Ernster, L.; Davies, K.J. The oxidative inactivation of mitochondrial electron transport chain components and ATPase. J. Biol. Chem. 1990, 265, 16330-16336.

22. Gardner, P.R.; Raineri, I.; Epstein, L.B.; White, C.W. Superoxide radical and iron modulate aconitase activity in mammalian cells. J. Biol. Chem. 1995, 270, 13399-13405, doi:10.1074/jbc.270.22.13399.

23. Gardner, P.R. Superoxide-driven aconitase FE-S center cycling. Biosci. Rep. 1997, 17, 33-42.

24. Case, A.J.; Madsen, J.M.; Motto, D.G.; Meyerholz, D.K.; Domann, F.E. Manganese superoxide dismutase depletion in murine hematopoietic stem cells perturbs iron homeostasis, globin switching, and epigenetic control in erythrocyte precursorcells. Free Radic. Biol. Med. 2013, 56, 17-27, doi:10.1016/j.freeradbiomed.2012.11.018. 
25. Benov, L. How superoxide radical damages the cell. Protoplasma 2001, 217, 33-36, doi:10.1007/BF01289410.

26. Wallace, M.A.; Liou, L.-L.; Martins, J.; Clement, M.H.S.; Bailey, S.; Longo, V.D.; Valentine, J.S.; Gralla, E.B. Superoxide Inhibits 4Fe-4S Cluster Enzymes Involved in Amino Acid Biosynthesis CROSS-COMPARTMENT PROTECTION BY CuZnSUPEROXIDE DISMUTASE. J. Biol. Chem. 2004, 279, 32055-32062, doi:10.1074/jbc.M403590200.

27. Li, Y.; Huang, T.-T.; Carlson, E.J.; Melov, S.; Ursell, P.C.; Olson, J.L.; Noble, L.J.; Yoshimura, M.P.; Berger, C.; Chan, P.H.; et al. Dilated cardiomyopathy and neonatal lethality in mutant mice lacking manganese superoxide dismutase. Nat. Genet. 1995, 11, 376, doi:10.1038/ng1295-376.

28. Cramer-Morales, K.; Heer, C.D.; Mapuskar, K.A.; Domann, F.E. SOD2 targeted gene editing by CRISPR/Cas9 yields Human cells devoid of MnSOD. Free Radic. Biol. Med. 2015, 89, 379-386, doi:10.1016/j.freeradbiomed.2015.07.017.

29. Melov, S.; Coskun, P.; Patel, M.; Tuinstra, R.; Cottrell, B.; Jun, A.S.; Zastawny, T.H.; Dizdaroglu, M.; Goodman, S.I.; Huang, T.-T.; et al. Mitochondrial disease in superoxide dismutase 2 mutant mice. Proc. Natl. Acad. Sci. 1999, 96, 846-851, doi:10.1073/pnas.96.3.846.

30. Case, A.J.; McGill, J.L.; Tygrett, L.T.; Shirasawa, T.; Spitz, D.R.; Waldschmidt, T.J.; Legge, K.L.; Domann, F.E. Elevated mitochondrial superoxide disrupts normal T cell development, impairing adaptive immune responses to an influenza challenge. Free Radic. Biol. Med. 2011, 50, 448-458, doi:10.1016/j.freeradbiomed.2010.11.025.

31. Dorn, I.; Lazar-Karsten, P.; Boie, S.; Ribbat, J.; Hartwig, D.; Driller, B.; Kirchner, H.; Schlenke, P. In vitro proliferation and differentiation of human CD34+ cells from peripheral blood into mature red blood cells with two different cell culture systems. Transfusion (Paris) 2008, 48, 1122-1132, doi:10.1111/j.15372995.2008.01653.x.

32. de Isla, N.G.; Riquelme, B.D.; Rasia, R.J.; Valverde, J.R.; Stoltz, J.F. Quantification of glycophorin A and glycophorin B on normal human RBCs by flow cytometry. Transfusion (Paris) 2003, 43, 1145-1152, doi:10.1046/j.15372995.2003.00471.x.

33. Pande, A.; Dorwal, P.; Jain, D.; Tyagi, N.; Mehra, S.; Sachdev, R.; Raina, V. Expression of CD71 by flow cytometry in acute leukemias: More often seen in acute myeloid leukemia. Indian J. Pathol. Microbiol. 2016, 59, 310-313, doi:10.4103/0377-4929.188145.

34. Marsee, D.K.; Pinkus, G.S.; Yu, H. CD71 (Transferrin Receptor)An Effective Marker for Erythroid Precursors in Bone Marrow Biopsy Specimens. Am. J. Clin. Pathol. 2010, 134, 429-435, doi:10.1309/AJCPCRK3MOAOJ6AT.

35. Wangen, J.R.; Brodersen, L.E.; Stolk, T.T.; Wells, D.A.; Loken, M.R. Assessment of normal erythropoiesis by flow cytometry: important considerations for specimen preparation. Int. J. Lab. Hematol. 2014, 36, 184-196, doi:10.1111/ijlh.12151.

36. Huang, Y.; Peng, J.; Oberley, L.W.; Domann, F.E. Transcriptional Inhibition of Manganese Superoxide Dismutase (SOD2) Gene Expression by DNA 
Methylation of the 5' CpG Island. Free Radic. Biol. Med. 1997, 23, 314-320, doi:10.1016/S0891-5849(97)00095-6.

37. Huang, Y.; He, T.; Domann, F.E. Decreased Expression of Manganese Superoxide Dismutase in Transformed Cells Is Associated with Increased Cytosine Methylation of the SOD2 Gene. DNA Cell Biol. 1999, 18, 643-652, doi:10.1089/104454999315051.

38. Hitchler, M.; Wikainapakul, K.; Yu, L.; Powers, K.; Attatippaholkun, W.; Domann, F. Epigenetic Regulation of Manganese Superoxide Dismutase Expression in Human Breast Cancer Cells. Epigenetics 2006, 1, 163-171, doi:10.4161/epi.1.4.3401.

39. Dhar, S.K.; Tangpong, J.; Chaiswing, L.; Oberley, T.D.; St Clair, D.K. Manganese superoxide dismutase is a p53-regulated gene that switches cancers between early and advanced stages. Cancer Res. 2011, 71, 6684-6695, doi:10.1158/0008-5472.CAN-11-1233.

40. Cyr, A.R.; Hitchler, M.J.; Domann, F.E. Regulation of SOD2 in Cancer by Histone Modifications and CpG Methylation: Closing the Loop Between Redox Biology and Epigenetics. Antioxid. Redox Signal. 2012, 18, 1946-1955, doi:10.1089/ars.2012.4850.

41. Killian, J.K.; Kim, S.Y.; Miettinen, M.; Smith, C.; Merino, M.; Tsokos, M.; Quezado, M.; Smith, W.I.; Jahromi, M.S.; Xekouki, P.; et al. Succinate Dehydrogenase Mutation Underlies Global Epigenomic Divergence in Gastrointestinal Stromal Tumor. Cancer Discov. 2013, 3, 648-657, doi:10.1158/2159-8290.CD-13-0092.

42. Mason, E.F.; Hornick, J.L. Succinate dehydrogenase deficiency is associated with decreased 5-hydroxymethylcytosine production in gastrointestinal stromal tumors: implications for mechanisms of tumorigenesis. Mod. Pathol. 2013, 26, 1492-1497, doi:10.1038/modpathol.2013.86.

43. Malik, F.; Santiago, T.; Bahrami, A.; Davis, E.; McCarville, B.; Newman, S.; Azzato, E.M.; Davidoff, A.M.; Brennan, R.; Ellison, D.W.; et al. Dedifferentiation in SDHDeficient Gastrointestinal Stromal Tumor: A Report With Histologic, Immunophenotypic, and Molecular Characterization. Pediatr. Dev. Pathol. 2019, 1093526619846222 , doi:10.1177/1093526619846222.

44. Røsland, G.V.; Dyrstad, S.E.; Tusubira, D.; Helwa, R.; Tan, T.Z.; Lotsberg, M.L.; Pettersen, I.K.N.; Berg, A.; Kindt, C.; Hoel, F.; et al. Epithelial to mesenchymal transition (EMT) is associated with attenuation of succinate dehydrogenase (SDH) in breast cancer through reduced expression of SDHC. Cancer Metab. 2019, 7, 6, doi:10.1186/s40170-019-0197-8.

45. Zhang, D.; Wang, W.; Xiang, B.; Li, N.; Huang, S.; Zhou, W.; Sun, Y.; Wang, X.; Ma, J.; Li, G.; et al. Reduced succinate dehydrogenase B expression is associated with growth and de-differentiation of colorectal cancer cells. Tumor Biol. 2013, 34, 2337-2347, doi:10.1007/s13277-013-0781-4.

46. Hughes, C.E.; Coody, T.K.; Jeong, M.-Y.; Berg, J.A.; Winge, D.R.; Hughes, A.L. Cysteine Toxicity Drives Age-Related Mitochondrial Decline by Altering Iron Homeostasis. Cell 2020, 180, 296-310.e18, doi:10.1016/j.cell.2019.12.035.

47. Weber, R.A.; Yen, F.S.; Nicholson, S.P.V.; Alwaseem, H.; Bayraktar, E.C.; Alam, M.; Timson, R.C.; La, K.; Abu-Remaileh, M.; Molina, H.; et al. Maintaining Iron 
Homeostasis Is the Key Role of Lysosomal Acidity for Cell Proliferation. Mol. Cell 2020, 77, 645-655.e7, doi:10.1016/j.molcel.2020.01.003.

48. Sulkowski, P.L.; Sundaram, R.K.; Oeck, S.; Corso, C.D.; Liu, Y.; Noorbakhsh, S.; Niger, M.; Boeke, M.; Ueno, D.; Kalathil, A.N.; et al. Krebs-cycle-deficient hereditary cancer syndromes are defined by defects in homologousrecombination DNA repair. Nat. Genet. 2018, 50, 1086-1092, doi:10.1038/s41588-018-0170-4.

49. Sulkowski, P.L.; Corso, C.D.; Robinson, N.D.; Scanlon, S.E.; Purshouse, K.R.; Bai, H.; Liu, Y.; Sundaram, R.K.; Hegan, D.C.; Fons, N.R.; et al. 2-Hydroxyglutarate produced by neomorphic IDH mutations suppresses homologous recombination and induces PARP inhibitor sensitivity. Sci. Transl. Med. 2017, 9, doi:10.1126/scitranslmed.aal2463.

50. Sulkowski, P.L.; Oeck, S.; Dow, J.; Economos, N.G.; Mirfakhraie, L.; Liu, Y.; Noronha, K.; Bao, X.; Li, J.; Shuch, B.M.; et al. Oncometabolites suppress DNA repair by disrupting local chromatin signalling. Nature 2020, 582, 586-591, doi:10.1038/s41586-020-2363-0.

51. Turcan, S.; Rohle, D.; Goenka, A.; Walsh, L.A.; Fang, F.; Yilmaz, E.; Campos, C.; Fabius, A.W.M.; Lu, C.; Ward, P.S.; et al. IDH1 mutation is sufficient to establish the glioma hypermethylator phenotype. Nature 2012, 483, 479-483, doi:10.1038/nature10866.

52. Noushmehr, H.; Weisenberger, D.J.; Diefes, K.; Phillips, H.S.; Pujara, K.; Berman, B.P.; Pan, F.; Pelloski, C.E.; Sulman, E.P.; Bhat, K.P.; et al. Identification of a CpG Island Methylator Phenotype that Defines a Distinct Subgroup of Glioma. Cancer Cell 2010, 17, 510-522, doi:10.1016/j.ccr.2010.03.017.

53. Lu, C.; Ward, P.S.; Kapoor, G.S.; Rohle, D.; Turcan, S.; Abdel-Wahab, O.; Edwards, C.R.; Khanin, R.; Figueroa, M.E.; Melnick, A.; et al. IDH mutation impairs histone demethylation and results in a block to cell differentiation. Nature 2012, 483, 474-478, doi:10.1038/nature10860.

54. Flavahan, W.A.; Drier, Y.; Johnstone, S.E.; Hemming, M.L.; Tarjan, D.R.; Hegazi, E.; Shareef, S.J.; Javed, N.M.; Raut, C.P.; Eschle, B.K.; et al. Altered chromosomal topology drives oncogenic programs in SDH-deficient GISTs. Nature 2019, 575, 229-233, doi:10.1038/s41586-019-1668-3.

55. Flavahan, W.A.; Drier, Y.; Liau, B.B.; Gillespie, S.M.; Venteicher, A.S.; StemmerRachamimov, A.O.; Suvà, M.L.; Bernstein, B.E. Insulator dysfunction and oncogene activation in IDH mutant gliomas. Nature 2016, 529, 110-114, doi:10.1038/nature16490.

56. Moran-Crusio, K.; Reavie, L.; Shih, A.; Abdel-Wahab, O.; Ndiaye-Lobry, D.; Lobry, C.; Figueroa, M.E.; Vasanthakumar, A.; Patel, J.; Zhao, X.; et al. Tet2 Loss Leads to Increased Hematopoietic Stem Cell Self-Renewal and Myeloid Transformation. Cancer Cell 2011, 20, 11-24, doi:10.1016/j.ccr.2011.06.001.

57. Thienpont, B.; Steinbacher, J.; Zhao, H.; D’Anna, F.; Kuchnio, A.; Ploumakis, A.; Ghesquière, B.; Van Dyck, L.; Boeckx, B.; Schoonjans, L.; et al. Tumour hypoxia causes DNA hypermethylation by reducing TET activity. Nature 2016, 537, 6368, doi:10.1038/nature19081.

58. Burr, S.; Caldwell, A.; Chong, M.; Beretta, M.; Metcalf, S.; Hancock, M.; Arno, M.; Balu, S.; Kropf, V.L.; Mistry, R.K.; et al. Oxygen gradients can determine 
epigenetic asymmetry and cellular differentiation via differential regulation of Tet activity in embryonic stem cells. Nucleic Acids Res. 2018, 46, 1210-1226, doi:10.1093/nar/gkx1197.

59. Zhao, B.; Yang, Y.; Wang, X.; Chong, Z.; Yin, R.; Song, S.-H.; Zhao, C.; Li, C.; Huang, H.; Sun, B.-F.; et al. Redox-active quinones induces genome-wide DNA methylation changes by an iron-mediated and Tet-dependent mechanism. Nucleic Acids Res. 2014, 42, 1593-1605, doi:10.1093/nar/gkt1090.

60. Shenoy, N.; Bhagat, T.; Nieves, E.; Stenson, M.; Lawson, J.; Choudhary, G.S.; Habermann, T.; Nowakowski, G.; Singh, R.; Wu, X.; et al. Upregulation of TET activity with ascorbic acid induces epigenetic modulation of lymphoma cells. Blood Cancer J. 2017, 7, e587, doi:10.1038/bcj.2017.65.

61. Minor, E.A.; Court, B.L.; Young, J.I.; Wang, G. Ascorbate Induces Ten-Eleven Translocation (Tet) Methylcytosine Dioxygenase-mediated Generation of 5Hydroxymethylcytosine $\$$. J. Biol. Chem. 2013, 288, 13669-13674, doi:10.1074/jbc.C113.464800.

62. Shenoy, N.; Bhagat, T.D.; Cheville, J.; Lohse, C.; Bhattacharyya, S.; Tischer, A.; Machha, V.; Gordon-Mitchell, S.; Choudhary, G.; Wong, L.-F.; et al. Ascorbic acidinduced TET activation mitigates adverse hydroxymethylcytosine loss in renal cell carcinoma. J. Clin. Invest. 2019, 129, 1612-1625, doi:10.1172/JCI98747.

63. Cimmino, L.; Dolgalev, I.; Wang, Y.; Yoshimi, A.; Martin, G.H.; Wang, J.; Ng, V.; Xia, B.; Witkowski, M.T.; Mitchell-Flack, M.; et al. Restoration of TET2 Function Blocks Aberrant Self-Renewal and Leukemia Progression. Cell 2017, 170, 10791095.e20, doi:10.1016/j.cell.2017.07.032.

64. Ge, G.; Peng, D.; Xu, Z.; Guan, B.; Xin, Z.; He, Q.; Zhou, Y.; Li, X.; Zhou, L.; Ci, W. Restoration of 5-hydroxymethylcytosine by ascorbate blocks kidney tumour growth. EMBO Rep. 2018, 19, e45401, doi:10.15252/embr.201745401. 